\title{
Emoji Discourse Strategy: Breaking The Communication Barrier Between Teachers and "Post- 00" College Students
}

\author{
${ }^{1}$ South China Normal University, Guangzhou, Guangdong, China. \\ ${ }^{2}$ Huizhou Health Sciences Polytechnic, Huizhou, Guangdong, China. \\ ${ }^{3}$ South China Normal University, Guangzhou, Guangdong, China. \\ ${ }^{4}$ South China Normal University, Guangzhou, Guangdong, China. \\ ${ }^{5}$ South China Normal University, Guangzhou, Guangdong, China. \\ a147969408@qq.com \\ b383101894@qq.com \\ c1697438236@qq.com \\ d742273287@qq.com \\ e123963303@qq.com
}

Yang Wenjin ${ }^{1, \mathrm{a}}$, Wang Jie, ${ }^{2, \mathrm{~b}}$, Fan Yanfen ${ }^{* 3, \mathrm{c}}$, Lin Manjia ${ }^{4, \mathrm{~d}}$, Zeng Zhaohao ${ }^{5, \mathrm{e}}$

\begin{abstract}
Emoji discourse is the "expression body language" of social networking. "Post-00" college students use emoji instead of words to express themselves on the Internet. One of the characteristics of their interpersonal communication is the popularity of online fighting pictures and Internet memes. The use of memes not only merely meets their needs that can help them to express most instinctively and emotionally, but also is an effective way of communicating and reducing pressure. Education is a two-way communication between teachers and students to transfer knowledge and cultivate talents. Teachers in colleges who are good at using emoji discourse strategies will effectively break the communication barriers with the "post-00" college students, and will be conducive to promote the growth of college students. It is the embodiment of the modern education concepts that keep pace with the times, which is of great significance to improve the quality of education.
\end{abstract}

Keywords: Emoji; College Teachers, "Post-00”, Breaking up, The Communication Barrier.

\section{THE ORIGIN OF THE PROBLEM: THE LOGICAL RELATIONSHIP BETWEEN EDUCATION AND COMMUNICATION}

Communication is of great significance in the process of teaching, and it plays an important role in the history of education. American educational philosopher John Dewey (1859-1952) emphasized in Democracy and Education, "Only by treating communication as a more basic and more lasting way of teaching, and then reflecting, can we put real teaching methods in place". The process of education is the process of life, and the process of life is the process of continuous communication leading to the transformation of experience. Therefore, all communication (and social life) is educational [1]. Education and communication have inherent rigorous logic. Education is communication [2], and education is a two-way communication between teachers and students to transfer knowledge and cultivate talents. According to Maslow's hierarchy of needs theory, with the development of social material and spiritual civilization, human beings increasingly need "respect needs and self-needs", and communication is a way for human feelings to reflect equality and respect both inside and outside. Therefore, communication is becoming increasingly important in the process of education in the new era. However, people's consciousness, thoughts and ideas are constantly updated under the development of the times, and consequently the communication barriers between teachers and college students also have new challenges. 


\section{OBSTACLE ANALYSIS: THE CONTRADICTION BETWEEN THE "HOT" PURSUIT AND THE "COOL" RESPONSE OF EMOJI}

As the society and the times change, people of different ages have great differences in values, knowledge, experience and personal characteristics, which leads to many communication barriers. At present, most of the teachers in Chinese universities are diligent, thrifty and eager to learn. They are well-behaved, not betraying their emotions at will. On the other hand, "post00 " college students have open individuality, natural and unrestrained. They dare to think and dare to do. They not only advocate the spirit of "Buddhism", which means enjoying the simple happiness without too much desires, but also insist the freedom of emotional expression, which means they like sending various emoji when chatting with others or expressing their opinions when watching videos. Today, with technological advancement and diversified access to information, "post-00" college students can express themselves in diversified ways. They can fully express themselves, show their personalities, and pay close attention to each other anytime and anywhere, discovering their own values and life platform in the weakly connected group. However, college teachers are mostly "before 90", who have experienced tremendous changes in Chinese society. Their growth background, growth environment and lifestyle are quite different from modern "post-00" college students. Therefore, the two often have conflict in views, concepts, personal expression and comprehension ability because of communication barriers. In class, teachers "act alone" on stage, while their students under the stage "work hard" on their phones. They completely ignore the content of the class, and play their phones undisturbedly. Some students even "modify" the photos of their teachers to make emoji, which can be used when students chat freely and playfully in the WeChat groups. In daily life, students just simply say hello when they meet their teachers, while some students even pretend to lower their head or make a phone call and then flee away when they bump into their teachers. What's more, the scene "the two with no fate may not know each other even when they are near each other" may appear. and the "fate" is the embodiment of communication. In addition to the main class, in secondary class such as ideology guidance, campus culture, and social practice, the effectiveness of education is not obvious due to insufficient communication. The "mechanical" teaching and guidance method is likely to dissatisfy "post- 00 " college students. Therefore, it is urgent to break the communication barriers between college teachers and "post-00" college students. From the perspective of emoji discourse strategy, the current communication barriers can be analyzed from two dimensions: the "hot" phenomenon of "post-00" college students' using emoji and college teachers' "cold" response.

\subsection{Post-00 College Students' Hot Pursuit of Emoji}

Following the popularity of internet language, various emoji (characters, pictures, animated pictures, pictures combined with texts, etc.) have "emerged suddenly" in cyberspace and social platforms, and become popular among netizens, especially among "post 00 ". Now emoji have become a universal and popular expression of internet discourse, and are frequently used in various occasions. Regarding emoji there are some funny expressions like "One set of emoji is omnipotent in communication. If it is not enough, use two", "Today no one dare to say he/she is good at communicating without emoji", etc [3]. This shows that the use of emoji has been integrated into the online social life of "post-00" college students. According to data released by China Youth Daily on July 18, 2017, more than 15,000 sets of emoji have been put on the WeChat emoji platform since it was launched in July 2015, and the users of WeChat use the platform for more than 600 million times a day. The development and popularity of emoji in the communication of the post- 00 is due to their psychological demands of personal development, as well as the specific social factors. In general, the reasons for the popularity of emoji discourse among "post-00" college students can be summarized into three aspects.

Firstly, emoji discourse brings personalized, popularized, convenient and entertaining discourse expression. There are various types of emoji, including cartoons, screenshots, real people and artistic creations. All kinds of emoji can express the user's personality, preferences and true feelings to the maximum extent. Emoji such as "pleasure, anger, sorrow, joy, embarrassment, boredom and helplessness" on WeChat can express the current emotional state of the users in a very popular way, making communication more effective. Emoji are very convenient to use. People can use those in social platforms such as QQ, WeChat, Weibo, and bilibili. They can also use other people's special creations in their favorites, buy a star series, or use their own creations, which can greatly improve the efficiency of communication and reduce the sense of rigidity and embarrassment caused by text-only communication. The emoji with text can achieve the effect of "entertaining in communication and communicating with entertainment". Due to these characteristics of emoji and the support of various network social platforms such as WeChat for emoji made by software such as Mei Tu Xiu Xiu, Photoshop and Flash. Emoji have become one of the important emotional carriers for the post-00 to express themselves and their personalities in a simple and quick way, deeply loved by the "post- 00 " college students. 
Secondly, emoji discourse caters to the needs of "post-00" college students to express their moods, emotions and thoughts. "Hilarity in imitation, acquisition of the sense of identity and resistance of discourse power are the reasons for netizens to use network emoji" [4]. "Post-00" college students are in adolescence of sentimentality. In this period, they are active in thinking and their psychological state and values are in the stage of formation and maturity. They are constantly exploring outwards to shape their own personality. Therefore, they are sensitive and they need to express their strong emotion. As mentioned before, emoji discourse is the "facial expression and body language" in the new era of internet social communication, which can be used as a typically nonverbal and visual way of expressing feelings and caters to the needs of the "post-00" to express emotions.

Thirdly, emoji discourse promotes relaxing and interesting social activities. Since most of the "post-00" college students grow up in the dual environment - family and school, they are not good at communicating in real life, and they can only rely on the internet to express their various demands. Therefore, the demands of social networking have changed accordingly and it has become more and more entertaining, interesting and grouporiented. They rely on the online appeal to find a relaxing and pleasant communication mode from the content, method and time of the chat to relieve their unhappiness and reduce various pressures. A study conducted in the past two years has found that at the night before the final exam, WeChat and QQ group of "post-00" college students will be extremely active and the emoji will "fly all over the screen" in order to reduce pre-exam stress and regulate emotions. Emoji discourse has the function of expression and body language, and more importantly, both sender and receiver can feel the "warmness" from it, which is the key reason why post-00s college students are keen on using emoji discourse.

\subsection{College Teachers" "Cold" Response to Emoji}

Since currently most of the college teachers are "before 90" and more are "before 85", they often take a "cold" attitude toward the use of emoji. Their knowledge acquisition and academic research are all accumulated through real hard work. Therefore, in teaching process, college teachers often combine their own learning and growth experiences to "instill" knowledge and ideas into students, which leads to a misalignment of the methods of knowledge acquisition and emotional transmission between teachers and "post-00" college students. It is not conducive to the realization of teaching effect and the development of educating. There are three main reasons why college teachers give "cold" responses to emoji discourse.

Firstly, they do not like it. The age and growth environment have caused college teachers not to use emoji to express their thoughts and feelings. They prefer to use real theories, data, and diagrams to explain something. For the emerging emoji discourses, they think that they are showy and not practical.

Secondly, they are not open enough. At present, most college teachers do not understand the "hot" degree of emoji discourse among "post-00" college students and are unwilling to spend more time on studying the culture of emoji. They also think that some teachers who use emoji to write comments to students "fail to do their jobs properly" and "spoil their students'. When their photos are "modified" as emoji by their students to express thoughts, emotions and love for them in the WeChat group, teachers will think that it is disrespectful and impolite to them and even infringes the right of portrait. Therefore, they will have misunderstandings and forbid the use of the photos by the students.

Thirdly, they are not good at it. In order to activate the classroom atmosphere and improve the quality of teaching, some teachers hope to use emoji in their teaching content. However, they fail under most circumstances. Some problems like the use of oldfashioned emoji or the misuse of them have caused students' misunderstanding of teaching semantics. "Since you don't use it well, then don't use it." Some teachers will respond with this attitude to the situations where the effect of initial use is not obvious.

\section{EMOJI DISCOURSE: AN EFFECTIVE STRATEGY TO BREAK THE COMMUNICATION BARRIERS BETWEEN COLLEGE TEACHERS AND "POST-00" COLLEGE STUDENTS}

Making good use of emoji plays an important role in breaking down the communication barriers between college teachers and "post-00" college students. It is conducive to promoting the whole process of education for college students in the new era. It is a manifestation of modern education and teaching concepts that keep pace with the times, and it is of great significance for college teachers to improve the quality of education.

\subsection{Understanding the Discourse of Online Communication in the New Era: Emoji Discourse is the "Facial Expression and Body Language" of Social Networking}

How do the facial expression and body language behave in the internet compared with those in the real life? The American communication scientist Albert Melabian once put forward the formula of the Melabian Communication Model for effective communication: total expression of information in communication $=$ words $(7 \%)+$ tone and intonation $(38 \%)+$ facial expression and body language (55\%) [5]. Melabian emphasized the scope of application of the formula, that 
is, it is not absolutely reflected in the proportion of the formula in any situation or occasion (for example, the formula cannot be applied to special populations such as deaf-mutes and blind people). The true meaning of the model is to clarify that effective communication is an allround matter, and body language and tone of voice play a great role in any effective communication. With the development of the internet and smart phones as well as the advent of the omni-media era, people's internet life occupies most of their overall life, resulting in less faceto-face communication and more online social networking. Even in the relationship between girlfriends, most of the partners also communicate through the internet, which is especially obvious among the "post-00" college students.

In online social communication, while "post-00" college students find it difficult to express emotions, thoughts and semantics without body language, the discourse function of emoji just realizes most of the functions of facial expressions and body language in face-to-face communication in real life. Looking back at the "evolutionary history" of emoji, it has now "evolved" to the "3.0 era". According to the way of presentation, it can be divided into two types: expressions with text and simple image expressions. According to content, it can also be divided into animal animation, exaggerated grassroots expressions, film and television screenshots, etc. According to emotions expressed, it can be divided into cute, cool, obscene, and exaggerated types and according to communication field, it can be divided into acquaintance expressions in the acquaintances and celebrity expressions in public areas [6]. It can be seen that in modern communication, emoji discourse has basically replaced the function of face-to-face body language in real life, and has important meaning and effect on effective communication that keeps pace with the times. Therefore, emoji discourse is the "facial expression and body language" of social networking.

\subsection{Changing Traditional Teaching Idea: Satisfying the Psychological Demands behind Students' Emoji Discourse}

At the National Conference on ideological and political work in Colleges and Universities, General Secretary $\mathrm{Xi}$ Jinping stressed that "new media and technologies should be applied to invigorate the work, to promote the high integration of the traditional advantages of ideological and political work with information technology, and to enhance the attraction and the sense of times [7]". New media has played a strong leading role in the innovation and development of education. It has had a significant impact on the communication style of "post00" college students and has changed the traditional educational methods of colleges and universities. The educational mode has changed from closed to open, from single to multiple. The use of emoji discourse also follow this trend. Ji Dahai, a well-known educational expert and researcher of the Sichuan Education Society, believes that using popular teaching methods among students, such as vivid cartoons, music, and videos, is what is pursued in the current reform and development of teaching methods [8]. At the same time, emoji have implication, which is regarded as implicit education in explicit teaching [9]. Therefore, college teachers should quickly change the traditional idea of education in order to meet the psychological demands behind the emoji of college students in the new era and effectively break down the communication barriers in education.

The "emoji test" which is supported and loved by the students is the best example. On December 11, 2017, the official account of the Guiyang Evening News published an article which was titled This "emoji test" is popular! The students said they liked it! The teacher said... The picture in that article was a special "emoji test" designed by a biology teacher of Guiyang National Middle School.

This biological paper with emoji vivified the boring biological knowledge of "hormonal regulation" with the use of emoji discourse. Some emoji with entertaining and ridiculous tone like "I am not happy", "Northerners rely on heating for winter while southerners rely on integrity for winter" were integrated into the content of "how to adjust body temperature", and interesting emoji were also attached according to the different situations in the exam. This way can not only make the test lively and interesting, but also relieve the pressure of students in senior three and deepen their thinking and consolidation of knowledge points. Some examinees said that "This kind of paper is very interesting and we can remember the knowledge better" "I want to come back to his class", and some students ridiculed that this is "the teacher of others". The paper using emoji has been fully recognized by students as well as affirmed and praised by the public. This is a reform of teaching methods. It represents the advance of teaching ideas and concepts as well as the progress and innovation of modern educational technology. Even so, it is worth emphasizing that not all stages of teaching are suitable for using emoji discourse. The application of emoji discourse should be scientific, rather than mechanical, to avoid the opposite effect.

\subsection{Making Good Use of Emoji Discourse to Communicate: Narrowing the Life and Psychological Distance between Teachers and Students}

"A teacher should impart knowledge and solve puzzles". They are the guardians and mentors on the path of students' growth. The ancients said: "Talent is the capital of virtue, and virtue is the commander of talent", which means virtue is indispensable for people' development. For students, possessing virtue and ability is the ideal goal. Therefore, teachers are not only disseminators of professional knowledge, but also 
educators of ideology, politics, moral quality, and mental health. They should take students' learning, life, social interaction and growth as their foothold and inspiration in the whole process of education. Therefore, the influence of teachers should not only be restricted to study, but also be extended to students' way of thinking and the principle of interpersonal communication, the basis of which is to establish a harmonious, pleasant and effective communication mode with students. At present, compared with face-to-face communication offline, "post-00" college students are more interested in online communication because of the characteristics of network communication such as invisibility and availability, and the processability of communication content, which satisfy the psychological and behavioral needs of contemporary teenagers, and affect the controllability of their self-expression and self-disclosure [10]. As a result, teachers who are good at using network communication naturally become the most effective way for the healthy and sustainable development of teacher-student relationship in the new era.

Teachers' using of emoji to communicate is a manifestation of their adeptness in network communication skills, and an important way to effectively shorten the life and psychological distance between teachers and students. If communication is the key to open people's minds, under the background that social networking has basically become the main part of communication, online communication is the silver key to open the minds of young teenagers in the internet age, while emoji discourses are the golden key. As stated before, emoji discourse is the "facial expression and body language" of social networking.

For teachers, using emoji to communicate with students is conducive to "friendly talk", which can change students' stereotypes that "the teacher gives orders while students only obey". If college students can interact with teachers by using emoji, especially those funny and special ones, it is a manifestation that both teachers and students can play jokes or respect and understand the emoji they send. At this time, students can easily open their minds and are willing to communicate with teachers and fully express their ideas; at the same time, students can also receive richer thoughts, ideas and other information from teachers through emoji. In short, only when the two parties of communication are established on the basis of equality of personality and status, can communication be carried out happily and effectively.

Teachers are good at using "thumbsup emoji" to communicate with students, which is an accelerator to integrate themselves into the student group. Tencent and Sina Weibo have played an irreplaceable role in promoting the use of emoji and enriching the content. According to the 2019 WeChat Data Report released by Tencent, the emoji like Facepalm, Grin, Chuckle,
ThumbsUp are the most frequently used "yellow face" emoji. In terms of the "Thumbsup" emoji, relatives and friends were often asked to give a like when this function was newly launched by WeChat. Later, giving a like has become a "habitual behavior" of everyone regardless of the photos and content in their circles. The more "like" people receive on their Moments, the more they will feel a sense of accomplishment. "ThumbsUp" is not only full of approval and appreciation, but also contains complex and diverse social emotions. Zhao Yawen and others from Tianjin Normal University studied the influence of WeChat users' "like" on their friends' circle, and believed that these behaviors could be divided into different motivational backgrounds according to their personality traits, among which "self-identity and social identity motivation" were the main motivational backgrounds [11]. Under the premise of laws and regulations, teachers can upload interesting content about study, work and life to the Moments. In addition to thumb up and leave some comments, students can also have a further understanding of their teachers' characters such as loveliness, naughty and sensibility, which can shorten the distance between teachers and students. Teachers thumb up and comment in students' circle of friends, which will also make students feel happy.

\subsection{The Development Trend of Emoji Discourse: Keeping a Friendly and Trusting Distance between Teachers and Students}

The development of emoji has gone through the initial stage of emoji, using ASCII codes to form abstract mimics, to the intermediate stage of emoji, using yellow small round face emoticons, and then to the advanced stage of emoji, using current popular quotations, movie stars, video screenshots, animations, and hot events as materials, with hilarious text to form dynamic or static pictures [12]. Only when teachers keep up with the development pace of online emoji, and take the initiative to understand students and the hot topics of student groups, can they keep their emoji discourse with the times. Like network buzzwords, emoji discourses are replaced quickly among students. They will change in stages according to political current events, hot social issues, television works, or popular topics among students, such as the emoji of "Amazing Girl" Fu Yuanhui, the emoji of complaining final review and exams, the anime emoji of Mashimaro and Doraemon, the emoji of network buzzwords "I don't care what you think. I only care what I think" and some photo emoji. Therefore, in the times where emoji discourses change so fast, in order to create a pleasant atmosphere for communication and educate students subconsciously, teachers should be good at observing students' lives, keeping up with the times, focusing on current political hotspots, television works as well as the popular topics among students, using emoji appropriately, which can satisfy students' psychological demands and help build a friendly and trusting 
relationship with students. Only in this way can teachers make communication happier and better, and make education more effective and subconscious.

\subsection{Grasping the True Meaning of Emoji: Guiding Students to Use Emoji Correctly}

Emoji are a symbol that producers use to express their feelings and attitudes. However, many emoji are illustrated with vulgar pictures and vulgar words, which challenge the netizens' baseline of morality and their cognitive standards. This kind of "dirty" emoji can be used to vent or satisfy people's various emotions, and can give the young unsophisticated students a momentary "pleasure". If young students stay in this environment for a long time, it is bound to challenge their baseline of social morality, influence their personality qualities, cause fragmented cognitive illusion and subvert their mainstream values, thus eroding their correct world outlook, view on life and values. Teachers should fully understand the true meaning of emoticons so as to judge students' psychological dynamics and value orientation, to choose the right method and guide students to grow into talents [11]. Specifically, the correct guidance for students can be listed as the following aspects. Firstly, strengthening students' ideological and moral cultivation. Creating a good atmosphere with noble character around the student group, and guiding the students to consciously resist "dirty" emoji. Secondly, helping students establish awareness of laws and regulations. Students should understand the regulation of using emoji, and the laws behind it, with the aim of using emoji discourses reasonably without violating laws and regulations. Thirdly, students should be guided to develop the habit of enjoying group life, maintain good interpersonal relationships, and be wary of the "shallow" social interaction behind "hot" emoji. Fourthly, students should be guided to understand the culture of emoji, figure out the deep meaning of emoji discourses and be alert to excessive entertainment and structuralism. Fifthly, students should be trained to develop their ability to recognize, participate in, and use media, with the aim of developing their rational thinking and encouraging them to speak up rationally in the multi-value network platform [13].

\section{CONCLUSION}

In the context of the rapid development of new media and the increasing proportion and weight of online discourse, "post- 00 " college students increasingly dislike and even deliberately escape from the context of traditional discourse and reject the means of expression in traditional discourse. Traditional discourse is now highlighted as the embarrassment and helplessness of "language transit". No matter how new media technology develops, it cannot change the phenomenon that human beings are social animals. Whether under consciousness or subconsciousness, people are fond of and even eager for social communication, especially for "post- 00 " college students, who are in the stage of "newborn calves are not afraid of tigers". They need more social communication to express their emotions and aspirations. Although communication is necessary between teachers and "post- 00 " college students, the two sides find it difficult to communicate, or even unable to communicate. The problem lies in the communication barrier here. Therefore, it is particularly effective to use emoji discourse strategies to break down the communication barriers between teachers and "post-00" college students. In most cases, body language is the most intuitive and effective expression compared with oral expressions. In the Internet world, in addition to video interaction, emoji discourse is a substitute for body language. In combination with the real-time chat context, emoji can present the body language of the other in the minds of the communication parties, effectively improving the communication effect.

\section{REFERENCES}

[1] Dewey. Democracy and Education, Wang Chengxu, trans. Beijing: People's Education Press, 1990.

[2] Ma Fei. Education as Communication: The Contemporary Interpretation of Dewey's Democracy and Education, Contemporary Education Sciences, 2018, pp. 3-10.

[3] Anon. Sending Emoji without Reasons--The Analysis of the Social Contact under Emoji [EB/OL]. http://www.taiwan.cn/xwzx/PoliticsNews/201712/t 20171215_11879524.htm.

[4] Wang Yaoyao. Research on the Generation and Dissemination of Internet Emoji from the Perspective of Meme Theory: A Case Study of Emoji of Online Drama "Qing Yu Nian" , Journal of News Research, 2020, pp. 5-66.

[5] Anon. Melabian Communication Model [EB/OL]. http://www.xphabit.com/article/4002.html.

[6] Zheng Manning. Research on the Popularity of Network Expression Package and Its Turning of Discourse Space, Editorial Friend, 2016, pp. 42-46.

[7] Zhang Shuo. The Ideological and Political Work Runs through the Whole Process of Education to Create A New Situation of the Development of China's Higher Education., People's Daily, 2016-1209(001)

[8] Shen Xingyi. Experts Praise that Teachers in Senior Three Designed Emoji Test, Chengdu Commercial Daily, 2016-12-18 (003).

[9] Song Defa. "Teaching beyond Words": Hidden Curriculum Delivered Through Lectures, Modern 
University Education, 2020, pp. 42-46+112-113.

[10] Ji Haiying. Impact of Network Communication on Teenagers' Psychosocial Development, Journal of Nanjing Normal University (Social Science Edition), 2012, pp. 109-115.

[11] Zhao Yawen, Gao Yanyan, Liu Tuo, Yang Haibo, Yan Guoli. The Influence of Personality Trait on Motivation of WeChat Like-behavior, Studies of Psychology and Behavior, 2017, pp. 270-275.

[12] Yang Jianhua. The Guidance and Norms of Emoji Culture, People's Tribune, 2018, pp. 140-141.

[13] Dong Yingying. Analysis on the Popularity of Emoji among College Students after 1995, Ideological and Theoretical Education, 2017, pp. 75-78. 